even today, he says he knows of no researcher who has been able to reliably insert genes into primary mouse TIL cells (some other scientists say it has been done). Other data he presented, including proof that the TIL cells would express TNF in sufficient quantities, were simply sketchy.

In part due to the paucity of supporting data, several RAC members were concerned that Rosenberg's TNF-TIL trial appeared to blur the line between fundamental research and human clinical experimentation and questioned its description as gene 'therapy'. As Nelson Wivel, the executive secretary of the RAC, puts it, "the question is: do you call things therapy when they are so experimental that they don't have a good chance of success?"

In Rosenberg's defence, Bruce Chabner, the director of the Division of Cancer Treatment, argues that even if the TNF is not being expressed at sufficient levels, the patients may get some therapeutic benefit from the TIL cells themselves, which attack tumours on their own. But other scientists disagree; patients are eligible for TNFTIL therapy only if they have already failed standard TIL therapy, they point out. More TIL cells that have already been proven not to work are unlikely to make a difference.

In part to answer its concerns, the RAC attached several restrictions to Rosenberg's protocol. One was that he could not inject the genetically modified cells unless they were expressing at least 100 picograms of TNF per ml ( $10^{\kappa}$ TIL cells) for 24 hours. But that level, it turns out, is at least an order of magnitude lower than necessary to shrink tumours. And in practice, Rosenberg has sometimes had trouble even reaching the RAC threshold. For the first TNF trial, he had to request special permission to average tests so as to get above the 100 picogram figure.

Even now, Rosenberg says, TNF expression in the trials varies from 100 to 1,200 picograms. Only a few patients show TNF expression at or above the 1,000 picogram level that he says the mouse models suggest is the minimum for efficacy. Rosenberg is hoping that the new vector may improve TNF expression by a factor of five. But he has as yet made no moves to halt the trials using the current TNF vector, despite the evidence that it has not worked as hoped - a point on which the board has challenged him.

Another concern of the board is whether Rosenberg's data shows that the gene-modified TIL cells are localizing to the tumours, and not ending up in the liver instead. TNF is extremely toxic and could, if expressed at sites other than the tumour, actually kill the patient before the cancer does. At the moment, the low level of TNF expression has avoided any toxicity problems, but the board has asked Rosenberg to supply new data to prove that the TNF is actually ending up at the tumour site. At the October meeting, Ronald Levy, chairman of the board and a Stanford University oncologist, noted that Rosenberg's data so far showed that TNF-TIL localization is "about a quarter of a magnitude lower than was projected". Given that and the TNF expression problem, he said, "I just think that it is premature and unscientific to proceed with a clinical trial based on the pre-clinical or preliminary data that has been developed."

In general, the board is concerned that Rosenberg is trying in humans what he has not yet perfected in the test tube. TNF expression, for example, can be observed easily in the laboratory, yet Rosenberg embarked on trials before the expression problems were resolved.

Some researchers attribute the problems in the TNF-TIL trials to Rosenberg's unrelenting determination to be at the forefront of the competitive field of gene therapy and his belief that studies in terminally ill patients take precedence over fine points of in vitro research. In 1990 Rosenberg lost the race to be first in gene therapy to colleagues at NIH, who were treating a rare immune deficiency. But by then Rosenberg was clearly committed to going forward with his trial. In the two years since then he has inserted TNF-modified TIL cells into nine patients.

Rosenberg refuses to reveal the fate of those patients - most are believed to have died -- until he publishes his results in a scientific journal. But his commitment to scientific standards has not kept him from publicizing, in everything from his new book, The Transformed Cell, to numerous press interviews, the fact that one of the patients is still alive. Whether the TNF experiment was actually responsible for that single remission is an open question.

One former collaborator (and now critic) believes that in the TNF TIL trials Rosenberg has "crossed the ethical line" in portraying research as therapy. Chabner concedes that in these trials "it's hard for us to say to a patient that you're getting a therapeutic level. The therapy issue is only part of the experiment. It may not work." But what you can prove with the trial, he says, is whether or not TNF gene transfer works in TIL cells. Noting that the patients in the study are terminally ill and have already failed other therapies, Chabner argues that the scientific value of the experiment is enough to justify the trials.

With the board of scientific counsellors in revolt, those arguments may no longer be enough. The board is expected to forward its list of questions to Rosenberg this week, and take up the issue again at its February meeting. Unless Rosenberg can provide them with better answers than he has over the past year, board members could make good on their threat to recommend that his TNF-TIL trials be halted.

Christopher Anderson

\section{CERN blames full moon for LEP problems}

Munich \& London. Dogs howl, the tide rushes in and mild-mannered men turn into werewolves. Now scientists at CERN, the European Laboratory for Particle Physics in Geneva, have explained another lunar trick.

Mysterious variations in the energy of particle beams circulating in the Large Electron Positron collider (LEP) have been an unresolvable puzzle for years. The problem initially was not serious, but as experiments such as those to determine the mass of the $Z^{0}$ required increasing beam accuracy, a persistent uncertainty of $10 \mathrm{MeV}$ became the dominant error in the LEP experiments.

Now CERN's particle physicists, along with colleagues from Stanford and Lausanne, finally have the answer. Tipped off by their

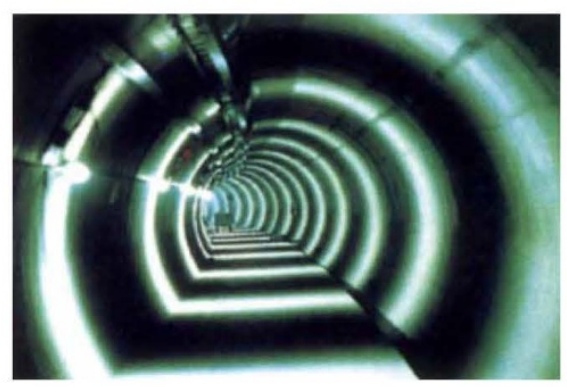

Driven round the bend: the LEP tunnel during the construction phase, before tidal effects had been considered.

California colleagues about the hazards of natural seismicity on beam energy, the LEP team realized that their variations were caused by minute deformations of the earth's crust due to the combined 'tidal' attraction of the sun and the moon. As the Moon passes overhead, it distorts the local radius of the Earth by $20 \mathrm{~cm}$, changing the $27 \mathrm{~km}$ length of the accelerator by all of $1 \mathrm{~mm}$. Because the number of revolutions per second of the particles in the beam is kept strictly constant, small changes in dimension obliges them to follow different orbits around the accelerator, which in turn either decreases or increases the beam energy.

The easiest cure, says Lyndon Evans of CERN, is to correct the data for the phases of the moon, although it could be possible to allow for tidal changes while experiments are running. That is easy enough; all LEP accelerator physicists have to remember now is to consult the phase of the moon - perhaps by checking the palms of their hands for hair — when calibrating beam energy.

Alison Abbott \& Roland Pease 